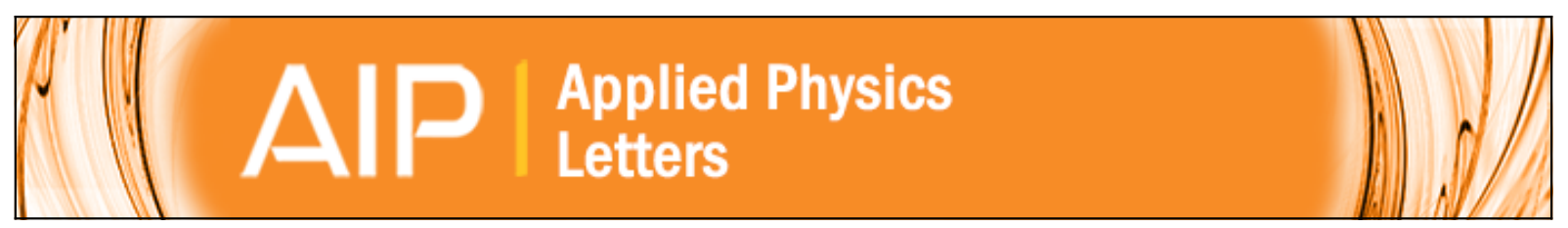

\title{
Fast generation of domain walls with defined chirality in nanowires
}

Kathrin Sentker, Falk-Ulrich Stein, Lars Bocklage, Toru Matsuyama, Mi-Young Im, Peter Fischer, and Guido Meier

Citation: Applied Physics Letters 104, 172404 (2014); doi: 10.1063/1.4874803

View online: http://dx.doi.org/10.1063/1.4874803

View Table of Contents: http://scitation.aip.org/content/aip/journal/apl/104/17?ver=pdfcov

Published by the AIP Publishing

\section{Articles you may be interested in}

Remote domain wall chirality measurement via stray field detection

J. Appl. Phys. 110, 123912 (2011); 10.1063/1.3671615

Currentinduced coupled domain wall motions in a twonanowire system

Appl. Phys. Lett. 99, 152501 (2011); 10.1063/1.3650706

Magnetic imaging of the pinning mechanism of asymmetric transverse domain walls in ferromagnetic nanowires Appl. Phys. Lett. 97, 233102 (2010); 10.1063/1.3523351

Controllable chirality switching of a moving domain wall by oblique magnetic field

Appl. Phys. Lett. 97, 032507 (2010); 10.1063/1.3467456

The effect of geometrical confinement and chirality on domain wall pinning behavior in planar nanowires J. Appl. Phys. 104, 033904 (2008); 10.1063/1.2961313

\section{A|P| $\left.\right|_{\text {Applied Physics }} ^{\text {Journal of }}$}




\title{
Fast generation of domain walls with defined chirality in nanowires
}

\author{
Kathrin Sentker, ${ }^{1, a)}$ Falk-Ulrich Stein, ${ }^{1}$ Lars Bocklage,${ }^{2,1,3}$ Toru Matsuyama, ${ }^{1}$ Mi-Young $\operatorname{Im},{ }^{4}$ \\ Peter Fischer, ${ }^{4}$ and Guido Meier ${ }^{1,3}$ \\ ${ }^{1}$ Institut für Angewandte Physik und Zentrum für Mikrostrukturforschung, Universität Hamburg, \\ Jungiusstr. 11, 20355 Hamburg, Germany \\ ${ }^{2}$ Deutsches Elektronen-Synchrotron, Notkestr. 85, 22607 Hamburg, Germany \\ ${ }^{3}$ The Hamburg Centre for Ultrafast Imaging, Luruper Chaussee 149, 22761 Hamburg, Germany \\ ${ }^{4}$ Center for X-ray Optics, Lawrence Berkeley National Laboratory, 1 Cyclotron Rd, Berkeley, \\ California 94720, USA
}

(Received 25 February 2014; accepted 23 April 2014; published online 1 May 2014)

We demonstrate the fast generation of domain walls with defined chiralities in nanowires. Nanosecond long current pulses are passed through a stripline to generate Oersted fields which create vortex domain walls in the nanowire. The direction of the Oersted field with respect to the nanowire is varied by the polarity of the voltage pulses as well by the alignment between the stripline and the nanowire. The results show that by using a tilted stripline and short current pulses domain walls with defined chirality and type can be generated. () 2014 AIP Publishing LLC. [http://dx.doi.org/10.1063/1.4874803]

Fast, energy efficient, and cheap nonvolatile magnetic memory devices are in the focus of magnetism research. ${ }^{1,2}$ The controllable motion of domain walls under the influence of spin polarized currents and magnetic fields has led to several ideas for applications like nonvolatile memory, logic devices, and sensors. ${ }^{2-4}$ The interest also extends to other fields of research like frustrated artificial spin ice systems. ${ }^{5}$ For fast domain wall based memories, the understanding of propagation and creation of domain walls in the nanosecond regime in nanowires is essential. ${ }^{2,6}$ One possibility to create domain walls in a nanowire is by an Oersted field resulting from a current pulse passing through a stripline perpendicular to the wire. ${ }^{7,8}$ This creation process has been studied as a dependence of the pulse amplitude and an external field. ${ }^{9}$ Also the threshold amplitude of the Oersted field for the domain wall creation, as a function of the applied field angle for non-perpendicular striplines was studied. ${ }^{10}$ But the effects of a non-perpendicular stripline on the domain-wall structure and especially its chirality, the sense of rotation of the magnetic moments inside the wall, have not been investigated yet. A controlled generation of domain walls with a defined chirality will be an important factor for creating reliable devices. For example, in artificial spin ice systems, the path of a vortex domain wall through the Y-shaped junctions depends on its sense of chirality. ${ }^{11,12}$

Here, we demonstrate the controlled generation of domain walls with defined chirality on a nanosecond timescale in permalloy $\left(\mathrm{Ni}_{80} \mathrm{Fe}_{20}\right)$ nanowires with a tilted stripline. The samples are prepared by two electron-beam lithography steps with positive PMMA resists and lift-off technique. First, the $8 \mu \mathrm{m}$ long, $20 \mathrm{~nm}$ thick, and $300 \mathrm{~nm}$ wide permalloy nanowires are prepared via thermal evaporation. In the second step, the $500 \mathrm{~nm}$ wide and $100 \mathrm{~nm}$ thick striplines are fabricated on top of the wire consisting of multiple material layers. To ensure good contact between the nanowire and the striplines, a layer sequence of $3 \mathrm{~nm}$ aluminium and $5 \mathrm{~nm}$ gold is deposited via sputter deposition after an in-situ argon

${ }^{a)}$ Electronic mail: ksentker@physnet.uni-hamburg.de plasma etching. Subsequently, using the same mask, $90 \mathrm{~nm}$ copper and $10 \mathrm{~nm}$ gold are deposited via thermal evaporation. The chirality is probed by breaking the symmetry of the wire via a notch and measuring the anisotropic magnetoresistance (AMR). ${ }^{13,14}$ The pinned domain walls at the notch show slightly different resistances that can be used to probe the wall structure. ${ }^{7}$ Notches with a depth of $130 \mathrm{~nm}$ are placed on one side of the wire. Figure 1(a) shows a scanning-electron micrograph of one sample as well as a schematic of the setup. The angle between the right stripline and the direction perpendicular to the nanowire is varied between $\pm 15^{\circ}, \pm 30^{\circ}$, and $\pm 45^{\circ}$ as shown in the schematic. The electron propagation direction is shown within the right stripline and downwards corresponds to negative voltage pulses. The $10 \mathrm{~ns}$ long voltage pulses for the creation of domain walls near the right stripline cause current pulses which divide between wire and stripline at the ratio of approximately $1: 10$ corresponding to the relation of their electric resistances. The pulse amplitudes are varied from $1 \mathrm{~V}$ to $4 \mathrm{~V}$ with negative and positive polarity, which result in Oersted fields from $20 \mathrm{mT}$ to $75 \mathrm{mT}$. Prior to every measurement, the wire is saturated in an external field of $100 \mathrm{mT}$ so that the magnetization of the wire and the Oersted field beneath the wire are aligned antiparallel. In addition to the voltage pulse, an external field is applied in $\mathrm{x}$-direction $(10 \mathrm{mT}$ to $-10 \mathrm{mT}$, see coordinate system, Fig. 1(a)) to investigate its influence on the domain-wall creation. Every combination of pulseand external field parameters is measured ten times. To detect and characterize the domain walls, the AMR is used (see Ref. 15). The resistance changes are used to detect the domain wall type (transverse or vortex) and their chirality. ${ }^{7}$ The nanowire dimensions are chosen to preferably create vortex walls. ${ }^{16}$ The resistance of the wire is measured by a small direct current of $100 \mu \mathrm{A}$ and all resistance changes are with respect to the resistance of the wire without a domain wall. Whether a head-to-head (hh) wall or a tail-to-tail (tt) wall is created depends on the initial magnetization of the wire. Head-to-head walls with positive (negative) chirality have the same magnetic pattern as tail-to-tail walls with 


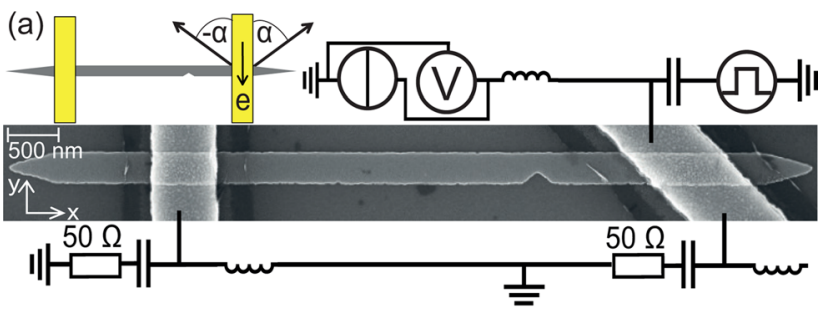

(b)

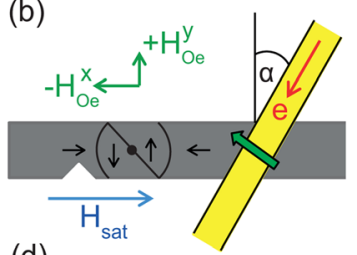

(d)

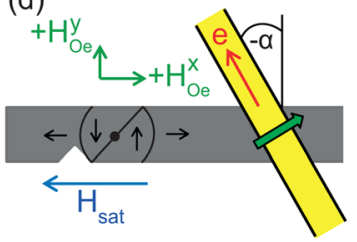

(e)
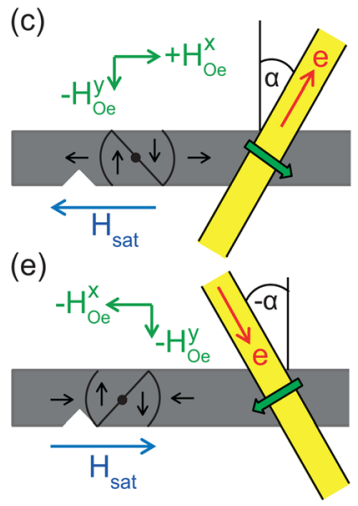

FIG. 1. (a) Schematic measurement setup and scanning electron micrograph of the nanowire with a notch and two crossing striplines. The current for Oersted field generation runs through the right stripline. (b) Depending on the stripline angle, the external field (blue) and the Oersted field (green) beneath the stripline (yellow), domain walls with different chiralities can be generated. Shown are also the $\mathrm{x}$ - and $\mathrm{y}$-components of the Oersted field. In (b) and (e), the situation for head-to-head walls and in (c) and (d) the situation for tail-to-tail walls is shown.

negative (positive) chirality and therefore yield the same resistance change when pinned at the notch. If both wall types have identical chiralities, they have a different magnetic pattern and therefore result in dissimilar resistance changes. The domain walls pin either at (Figs. 1(d) and 1(e)) or to the right of the notch (Figs. 1(b) and 1(c)). For example, the hh wall with a clockwise chirality pins at the notch (see Fig. 1(e)), whilst the hh wall with a counterclockwise chirality pins at the right side of the notch (see Fig. 1(b)). This pinning behaviour is related to the minimisation of the stray field at the notch. The difference in the measured resistance change is a result of varying perpendicular magnetization components along the current paths and is therefore larger if a hh wall with a clockwise chirality is present than in the case of a hh wall with a counterclockwise chirality. ${ }^{7}$ Because of the inversion symmetry, the behaviour is the opposite for tt walls (Figs. 1(c) and 1(d)). Figure 2 shows results for a stripline angle of $\pm 15^{\circ}$, which yields the best control of chirality when compared to the results for $\pm 30^{\circ}$ and $\pm 45^{\circ}$ (not shown). In Fig. 2, the counts of the number of generated domain walls for every resistance change level depending on the angle and pulse polarity are plotted. The diagram indicates that a negative stripline angle generates vortex walls with the larger resistance change $\Delta R_{2}$ irrespective of the pulse polarity. In concordance, a positive stripline angle generates vortex walls with the smaller resistance change $\Delta R_{1}$ again irrespective of the pulse polarity. Due to slightly different pinning of the domain wall at the notch and noise from the instruments, the resistance changes fluctuate. To quantitatively determine the various resistance changes, a Gaussian fit for every maximum is performed with amplitude, width, and resistance change of the particular

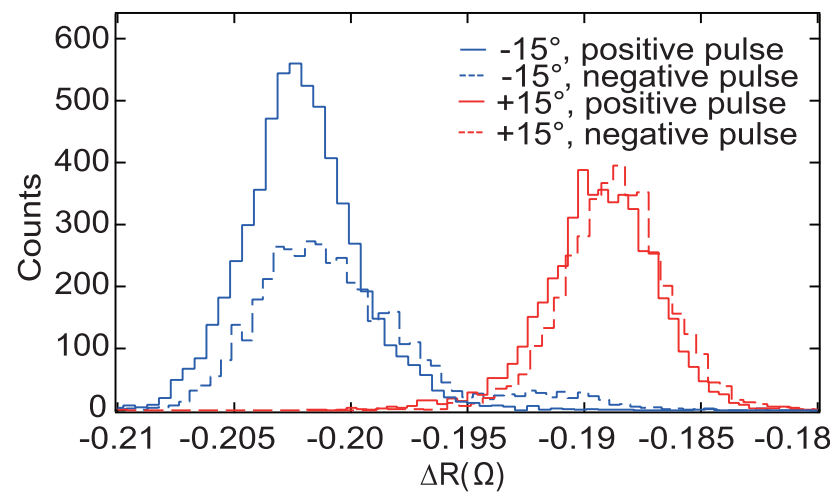

FIG. 2. Measurement of resistance changes due to domain wall generation for two angles between stripline and nanowire $\left( \pm 15^{\circ}\right)$ as well as positive and negative pulse polarity.

maximum resulting in $\Delta R_{1}=(-0,1891 \pm 0,0004) \Omega$ and $\Delta R_{2}=(-0,2021 \pm 0,0006) \Omega$. When the angle of the stripline is negative, there are only hh walls with a clockwise chirality and tt walls with counterclockwise chirality and vice versa if it is positive. To validate this correlation, we imaged the domain walls in our nanowires with full field soft X-ray transmission microscopy at the Advanced Light Source in Berkeley, CA, USA. ${ }^{17,18}$ The images were recorded at the Fe $\mathrm{L} 3$ edge $(707 \mathrm{eV})$ with a spatial resolution of about $25 \mathrm{~nm}$. Figure 3(a) shows a direct image with two

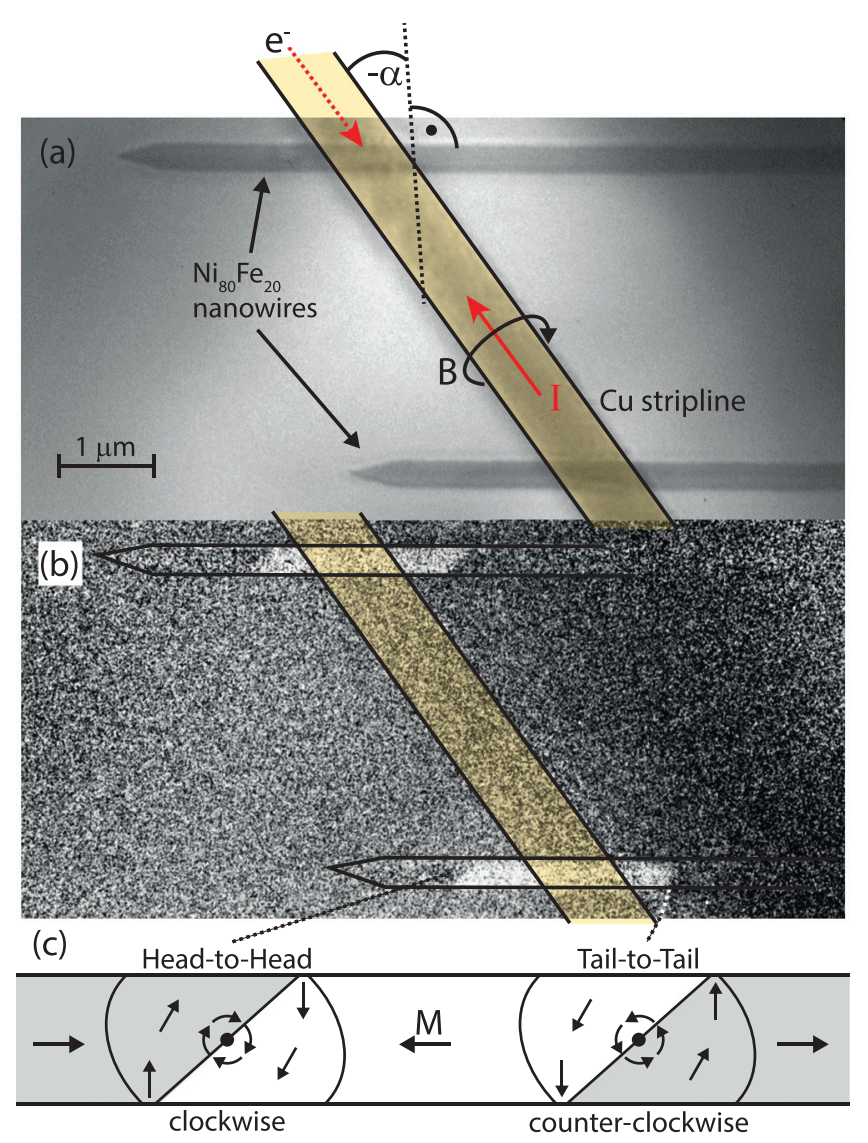

FIG. 3. (a) Transmission X-ray micrograph of two permalloy nanowires with a crossing copper stripline. (b) Differential image of the saturated- and the post current-pulse state. Domain walls are created on both sides of the stripline (transitions from black to white). The occurring magnetization configuration is shown in (c). 
TABLE I. Summary of the domain wall structure depending on the angle and pulse polarity. Tail-to-tail wall (tt) or head-to-head wall (hh) with a clockwise (cw) or counterclockwise (ccw) chirality. Indicated are the resistance changes which arise from the particular wall, whereby $\Delta R_{2}>\Delta R_{1}$.

\begin{tabular}{lrr}
\hline \hline & Positive angle & Negative angle \\
\hline Positive pulse & $\mathrm{tt}, \mathrm{cw} \Rightarrow \Delta R_{1}$ & $\mathrm{tt}, \mathrm{ccw} \Rightarrow \Delta R_{2}$ \\
Negative pulse & $\mathrm{hh}, \mathrm{ccw} \Rightarrow \Delta R_{1}$ & $\mathrm{hh}, \mathrm{cw} \Rightarrow \Delta R_{2}$ \\
\hline \hline
\end{tabular}

$300 \mathrm{~nm}$ wide and $20 \mathrm{~nm}$ thick permalloy nanowires without notches and a tilted copper stripline $\left(30^{\circ}\right)$ with an optimized layout for x-ray microscopy. The electron flow of the $10 \mathrm{~ns}$ long pulses is downwards. The situation on the left side is comparable to the one in Fig. 1(e) with a negative tilting of the stripline and an electron flow downwards. The layout on the right side is comparable to that in Fig. 1(d) when rotated by $180^{\circ}$. To enhance the magnetic contrast, an image of the saturated state is subtracted from an image after the pulse has been applied. The differential image is shown in Fig. 3(b). On both sides of the stripline, domain walls are created as indicated by the transitions from black to white. The occurring domain-wall structure is determined by the angle of the transitions and is displayed in Fig. 3(c). On the left side, a clockwise hh wall and on the right side a counterclockwise tt wall is created. This is in agreement with the electric measurements shown in Fig. 2.

These results can be understood by looking at the $\mathrm{x}$ and $y$-components of the Oersted field of the current pulses as depicted in Figs. 1(b)-1(e). The type (hh or tt) of the domain wall is determined by the initial magnetization. For domain wall creation, an antiparallel x-component of the Oersted field, compared to the initial magnetization, is necessary which corresponds to a certain pulse polarity. On the other hand, the chirality of the wall is controlled by the y-component which depends on the stripline angle and the pulse polarity. For example, a negative angle and a negative pulse (Fig. 1(e)) result in a negative $\mathrm{x}$-component as well as a negative y-component of the Oersted field. The negative $\mathrm{x}$-component generates an hh wall and the negative y-component a clockwise chirality. This wall has the same pattern as the tt wall with counterclockwise chirality in Fig. 1(d) resulting in the same resistance change $\Delta R_{2}$ for both cases. The four possible configurations of the angle and the pulse polarity (see Table I) allow us to inject a domain wall with defined chirality and type into the nanowire. To show the dependence of the chirality on the pulse amplitude and the applied external magnetic field, a statistical analysis of the individual pulse polarity/angle configurations of the electrical measurements in Fig. 2 is performed. Figure 4 shows the probabilities (color scale) that a vortex domain wall is present after a pulse depending on the external field and pulse amplitude. The regime in which a wall generation is most likely ranges from $+(-) 4 \mathrm{mT}$ to $-(+) 2 \mathrm{mT}$ and the pulse amplitude must be at least (less than) about $+(-) 1.9 \mathrm{~V}$ which corresponds to an Oersted field of $38 \mathrm{mT}$. The results in Figs. 4(a)-4(d) give asymmetric domain-wall generation probabilities. For example, Fig. 4(a) shows that the probability decreases with higher negative background fields, while the probability is zero for positive external fields higher than $+3 \mathrm{mT}$. The reason for this asymmetry is due to the notch. Up to field values of $-8 \mathrm{mT}$, the wall is pinned at the notch, whilst at a field value of $+3 \mathrm{mT}$, the two generated walls annihilate each other. No change in the resistance level is then measured. Neither the external field nor the amplitude
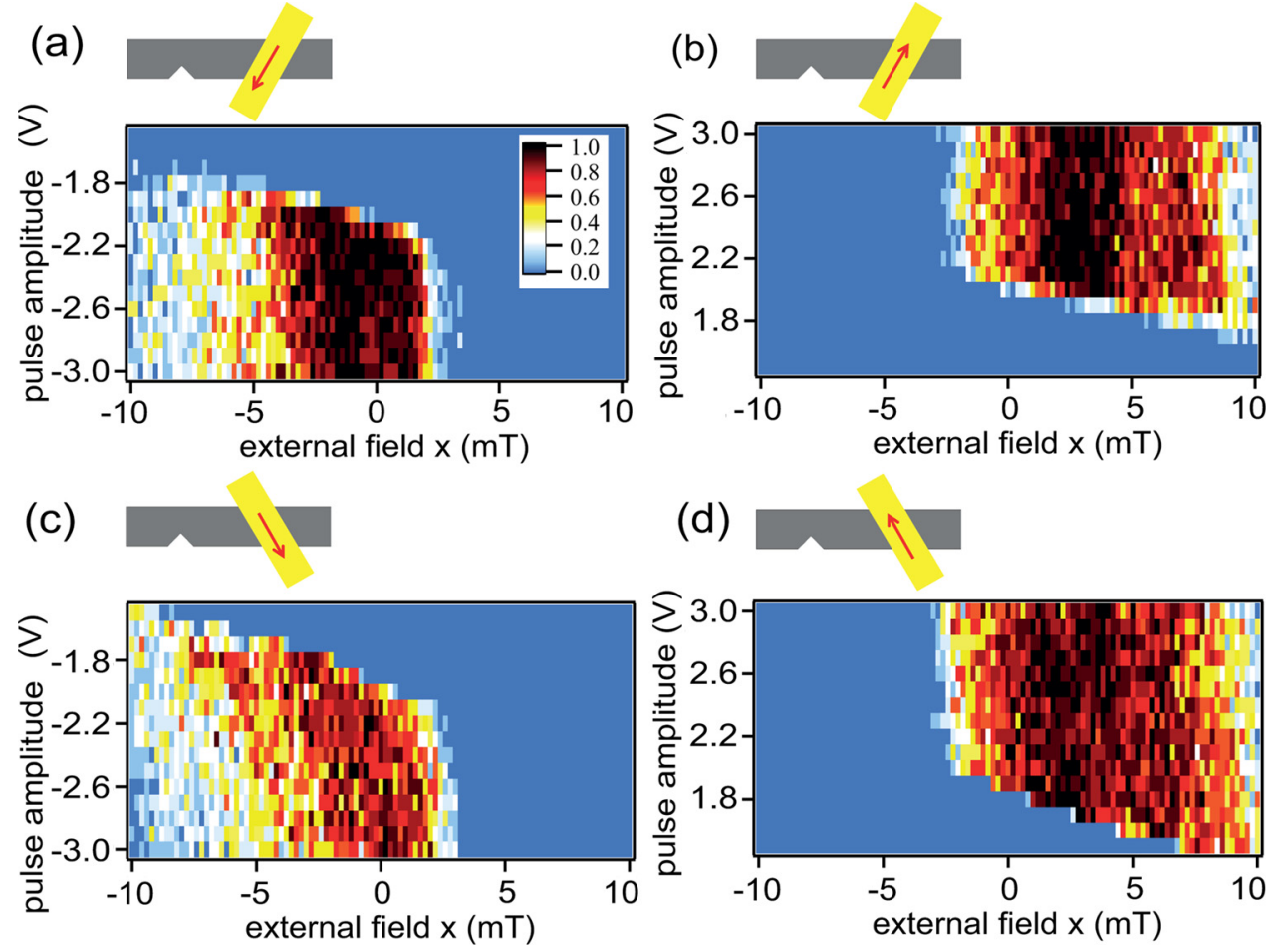

FIG. 4. Statistical analysis of domain wall generation. Shown are the probabilities (color scale) that a domain wall is present depending on the pulse and external field for $+15^{\circ}$ with negative pulse (a) and positive pulse (b) and for $-15^{\circ}$ with negative pulse (c) and positive pulse (d). 
have shown an impact on the sense of chirality of the created domain walls.

In summary, we demonstrated that a controlled generation of domain walls with a defined chirality is possible by changing the direction of the Oersted field. Various samples with different angles between stripline and nanowire have been investigated. The results show that a negative angle results in a hh wall with a clockwise chirality and a tt wall with a counterclockwise chirality and vice versa if the angle is positive. These results show that using a tilted stripline and nanosecond current pulses a fast generation of domain walls with defined type and chirality can be achieved. The general setup would allow for implementation in current driven devices as well as nanowire spin ice systems similar to Refs. 2 and 11. Multiple consecutively injected domain walls would show the same pattern regardless of the domainwall type increasing the reliability and predictability of their motion in wire networks.

We thank Ulrich Merkt for persistent support. Financial Support by the Deutsche Forschungsgemeinschaft via Sonderforschungsbereich 688 and via Graduiertenkolleg 1286 is gratefully acknowledged. This work was supported by the excellence cluster "The Hamburg Centre of Ultrafast Imaging-Structure, Dynamics, and Control of Matter on the Atomic Scale" of the Deutsche Forschungsgemeinschaft. This work was also supported by the Director, Office of Science, Office of Basic Energy Sciences, of the U.S. Department of Energy under Contract No. DE-AC0205CH11231 and the Leading Foreign Research Institute Recruitment Program (Grant No. 2012K1A4A3053565) through the National Research Foundation of Korea (NRF) funded by the Ministry of Education, Science and Technology (MEST).

${ }^{1}$ M. H. Kryder and C. S. Kim, IEEE Trans. Magn. 45, 3406 (2009).

${ }^{2}$ S. S. P. Parkin, M. Hayashi, and L. Thomas, Science 320, 190 (2008).

${ }^{3}$ D. A. Allwood, G. Xiong, and R. P. Cowburn, Appl. Phys. Lett. 85, 2848 (2004).

${ }^{4}$ R. Mattheis, S. Glathe, M. Diegel, and U. Hübner, J. Appl. Phys. 111, 113920 (2012).

${ }^{5}$ R. F. Wang, C. Nisoli, R. S. Freitas, J. Li, W. McConville, B. J. Cooley, M. S. Lund, N. Samarth, C. Leighton, V. H. Crespi, and P. Schiffer, Nature 439, 303 (2006).

${ }^{6}$ M. Hayashi, L. Thomas, R. Moriya, C. Rettner, and S. S. P. Parkin, Science 320, 209 (2008).

${ }^{7}$ M. Hayashi, L. Thomas, C. Rettner, R. Moriya, X. Jiang, and S. S. P. Parkin, Phys. Rev. Lett. 97, 207205 (2006).

${ }^{8}$ F.-U. Stein, L. Bocklage, M. Weigand, and G. Meier, Sci. Rep. 3, 1737 (2013).

${ }^{9}$ F.-U. Stein, L. Bocklage, T. Matsuyama, and G. Meier, Appl. Phys. Lett. 100, 192403 (2012).

${ }^{10}$ L. O Brien, D. E. Read, D. Petit, and R. P. Cowburn, J. Phys.: Condens. Matter 24, 024222 (2012)

${ }^{11}$ K. Zeissler, S. K. Walton, S. Ladak, D. E. Read, T. Tyliszczak, L. F. Cohen, and W. R. Branford, Sci. Rep. 3, 1252 (2013).

${ }^{12}$ A. Pushp, T. Phung, C. Rettner, B. P. Hughes, S.-H. Yang, L. Thomas, and S. S. P. Parkin, Nat. Phys. 9, 505 (2013).

${ }^{13}$ M. Kläui, C. A. F. Vaz, J. A. C. Bland, W. Wernsdorfer, G. Faini, E. Cambril, L. J. Heyderman, F. Nolting, and U. Rüdiger, Phys. Rev. Lett. 94, 106601 (2005).

${ }^{14}$ A. Himeno, T. Okuno, S. Kasai, T. Ono, S. Nasu, K. Mibu, and T. Shinjo, J. Appl. Phys. 97, 66101 (2005).

${ }^{15}$ G. Nahrwold, L. Bocklage, J. Scholtyssek, T. Matsuyama, B. Krüger, U. Merkt, and G. Meier, J. Appl. Phys. 105, 07D511 (2009).

${ }^{16}$ Y. Nakatani, A. Thiaville, and J. Miltat, J. Magn. Magn. Mater. 290, 750 (2005).

${ }^{17}$ D.-H. Kim, P. Fischer, W. Chao, E. Anderson, M.-Y. Im, S.-C. Shin, and S.-B. Choe, J. Appl. Phys. 99, 08 H303 (2006).

${ }^{18}$ L. Bocklage, B. Krüger, R. Eiselt, M. Bolte, P. Fischer, and G. Meier, Phys. Rev. B 78, 180405 (2008). 